\title{
Correction to: Cyber Polygon Site Project in the Framework of the MEPhI Network Security Intelligence Center
}

\author{
Natalia Miloslavskaya (D) and Alexander Tolstoy (D)
}

\section{Correction to:}

Chapter "Cyber Polygon Site Project in the Framework of the MEPhI Network Security Intelligence Center" in: A. V. Samsonovich et al. (Eds.): Brain-Inspired Cognitive Architectures for Artificial Intelligence: BICA*AI 2020, AISC 1310, https://doi.org/10.1007/978-3-030-65596-9_36

The original version of the book was inadvertently published without the DOI number in Reference 16 of Chapter 36. The DOI number has now been added along with the link https://urldefense.proofpoint.com/v2/url?u=https-3A_doi.org_10.1016_j.procs. 2020.02.113\&d=DwIF3g\&c=vh6FgFnduejNhPPD0fl_yRaSfZy8CWbWnIf4XJhSqx8 \&r=jeZ5gAbQde8tqHt7czlL8XpTksv3kwFGvx9LDEhGmBU\&m=IZa0e58gsmrRtS_ vyDKa0UjUdiqjWlaLWvooAzlX4nQ\&s=7GbuI1uwn03OqSFs7TFQd-R1QXcZvTw P6-1R8uGJ42Q\&e=. 